\title{
BMJ Open Patient-physician discordance in assessment of adherence to inhaled controller medication: a cross-sectional analysis of two cohorts
}

Cristina Jácome (D) , ${ }^{1}$ Ana Margarida Pereira, ${ }^{1,2}$ Rute Almeida (D) ${ }^{1}$ Manuel Ferreira-Magalhaes, ${ }^{1,3}$ Mariana Couto, ${ }^{2}$ Luís Araujo, ${ }^{2}$ Mariana Pereira, ${ }^{4}$ Magna Alves Correia, ${ }^{2}$ Cláudia Chaves Loureiro, ${ }^{5}$ Maria Joana Catarata, ${ }^{5}$ Lília Maia Santos, ${ }^{5}$ João Pereira, ${ }^{5}$ Bárbara Ramos, ${ }^{5}$ Cristina Lopes,, 6 Ana Mendes, ${ }^{8}$ José Carlos Cidrais Rodrigues, ${ }^{9}$ Georgeta Oliveira, ${ }^{9}$ Ana Paula Aguiar, ${ }^{9}$ Ivete Afonso, ${ }^{9}$ Joana Carvalho, ${ }^{9}$ Ana Arrobas, ${ }^{10}$ José Coutinho Costa, ${ }^{10}$ Joana Dias, ${ }^{10}$ Ana Todo Bom, ${ }^{11}$ João Azevedo, ${ }^{11}$ Carmelita Ribeiro, ${ }^{11}$ Marta Alves, ${ }^{11}$ Paula Leiria Pinto, ${ }^{12}$ Nuno Neuparth, ${ }^{12,13}$ Ana Palhinha, ${ }^{12}$ João Gaspar Marques, ${ }^{12}$ Nicole Pinto, ${ }^{12}$ Pedro Martins, ${ }^{12,13}$ Filipa Todo Bom, ${ }^{14}$ Maria Alvarenga Santos, ${ }^{14}$ Alberto Gomes Costa, ${ }^{15}$ Armandina Silva Neto, ${ }^{15}$ Marta Santalha, ${ }^{15}$ Carlos Lozoya, ${ }^{16}$ Natacha Santos, ${ }^{17}$ Diana Silva, ${ }^{18}$ Maria João Vasconcelos, ${ }^{18}$ Luís Taborda-Barata (1),${ }^{19,20}$ Célia Carvalhal, ${ }^{20}$ Maria Fernanda Teixeira, ${ }^{3}$ Rodrigo Rodrigues Alves, ${ }^{21}$ Ana Sofia Moreira, ${ }^{21}$ Cláudia Sofia Pinto, ${ }^{22}$ Pedro Morais Silva, ${ }^{23}$ Carlos Alves, ${ }^{24}$ Raquel Câmara, ${ }^{24}$ Didina Coelho, ${ }^{24}$ Diana Bordalo, ${ }^{25}$ Ricardo M Fernandes, ${ }^{26,27}$ Rosário Ferreira, ${ }^{26}$ Fernando Menezes, ${ }^{28}$ Ricardo Gomes, ${ }^{28}$ Maria José Calix, ${ }^{29}$ Ana Marques, ${ }^{29}$ João Cardoso, ${ }^{30,31}$ Madalena Emiliano, ${ }^{30}$ Rita Gerardo, ${ }^{30}$

To cite: Jácome C, Pereira AM, Almeida R, et al. Patientphysician discordance in assessment of adherence to inhaled controller medication: a cross-sectional analysis of two cohorts. BMJ Open 2019;9:e031732. doi:10.1136/ bmjopen-2019-031732

\section{- Prepublication history and} additional material for this paper are available online. To view please visit the journal (http:// dx.doi.org/10.1136/bmjopen2019-031732).

Received 17 May 2019 Revised 13 September 2019 Accepted 30 September 2019

\section{Check for updates}

(c) Author(s) (or their employer(s)) 2019. Re-use permitted under CC BY-NC. No commercial re-use. See rights and permissions. Published by BMJ.

For numbered affiliations see end of article.

Correspondence to

Dr Joao A Fonseca;

fonseca.ja@gmail.com

Carlos Nunes, ${ }^{32}$ Rita Câmara, ${ }^{33}$ José Alberto Ferreira ${ }^{34}$ Aurora Carvalho, ${ }^{34}$ Paulo Freitas, ${ }^{35}$ Ricardo Correia, ${ }^{36}$ Joao A Fonseca, ${ }^{1,2,4,36}$ Inspirers group

\section{ABSTRACT}

Objective We aimed to compare patient's and physician's ratings of inhaled medication adherence and to identify predictors of patient-physician discordance.

Design Baseline data from two prospective multicentre observational studies.

Setting 29 allergy, pulmonology and paediatric secondary care outpatient clinics in Portugal.

Participants 395 patients ( $\geq 13$ years old) with persistent asthma.

Measures Data on demographics, patient-physician relationship, upper airway control, asthma control, asthma treatment, forced expiratory volume in one second $\left(\mathrm{FEV}_{1}\right)$ and healthcare use were collected. Patients and physicians independently assessed adherence to inhaled controller medication during the previous week using a $100 \mathrm{~mm}$ Visual Analogue Scale (VAS). Discordance was defined as classification in distinct VAS categories (low 0-50; medium 51-80; high 81-100) or as an absolute difference in VAS scores $\geq 10 \mathrm{~mm}$. Correlation between patients' and physicians' VAS scores/categories was explored. A multinomial logistic regression identified the predictors of physician overestimation and underestimation.

Results High inhaler adherence was reported both by patients (median (percentile 25 to percentile 75) 85 (65-95) mm; 53\% VAS>80) and by physicians (84 (68-95) $\mathrm{mm} ; 53 \% \mathrm{VAS}>80$ ).
Correlation between patient and physician VAS scores was moderate $\left(r_{s}=0.580 ; p<0.001\right)$. Discordance occurred in $56 \%$ of cases: in $28 \%$ physicians overestimated adherence and in $27 \%$ underestimated. Low adherence as assessed by the physician ( $\mathrm{OR}=27.35$ (9.85 to 75.95$)), \mathrm{FEV}_{1} \geq 80 \%$ (OR=2.59 (1.08 to 6.20)) and a first appointment ( $\mathrm{OR}=5.63$ (1.24 to 25.56)) were predictors of underestimation. An uncontrolled asthma $(\mathrm{OR}=2.33$ (1.25 to 4.34)), uncontrolled upper aimay disease $(\mathrm{OR}=2.86$ (1.35 to 6.04)) and prescription of short-acting betaagonists alone ( $\mathrm{OR}=3.05$ (1.15 to 8.08)) were associated with overestimation. Medium adherence as assessed by the physician was significantly associated with higher risk of discordance, both for overestimation and underestimation of adherence $(\mathrm{OR}=14.50$ (6.04 to 34.81); $\mathrm{OR}=2.21$ (1.07 to 4.58)), while having a written action plan decreased the likelihood of discordance $(\mathrm{OR}=0.25$ (0.12 to 0.52$) ; 0 R=0.41$ (0.22 to 0.78$))\left(R^{2}=44 \%\right)$.

Conclusion Although both patients and physicians report high inhaler adherence, discordance occurred in half of cases. Implementation of objective adherence measures and effective communication are needed to improve patient-physician agreement.

\section{BACKGROUND}

Inhaled controller medications are the cornerstone of effective asthma treatment, ${ }^{1}$ 


\section{Strengths and limitations of this study}

Data from two multicentre studies with a similar design conducted at 29 secondary care outpatient clinics.

- Estimates of inhaled medication adherence were only based on Visual Analogue Scales, but these simple measures could be easily implemented during medical visits.

- Patient and physician estimates of adherence were not compared with objective data and were assessed only at one time-point.

- Predictors of discordance were identified in a multinomial model, but results may not be generalisable as patients were recruited by convenience sampling.

with established benefits in decreasing severity and frequency of symptoms as well as exacerbations. ${ }^{2}{ }^{3}$ However, to achieve these benefits, daily adherence to the prescribed inhaled medications is of critical importance.

Adherence rates in patients with asthma are known to be low, both in paediatric and adult studies. ${ }^{45}$ Suboptimal adherence to inhaled medication is associated with poor health outcomes, including lack of symptom control, exacerbations, emergency department visits and hospitalisations, leading to disease progression, additional social burden and health costs. ${ }^{6}$ To improve these health outcomes, it is crucial to promptly identify poorly adherent patients during medical visits, ${ }^{7}$ enabling physicians to address adherence barriers early and avoiding unnecessary additional diagnostic procedures and adjustments in medication. However, this is quite challenging as there is no commonly accepted approach to assess adherence.

Distinct methods have been used, such as evaluation of medical/dispensing records, use of electronic monitoring devices and reliance on self-reports. ${ }^{8-10}$ The first two methods have limited feasibility for routine use in clinical practice and resource-constrained settings. Selfreports, although subjective, are still considered one of the preferred methods to continuously monitor adherence as they are simple, cheap and minimally intrusive. ${ }^{112}$ One example is the use of a single item Visual Analogue Scale (VAS), which has shown to provide estimates of adherence comparable with pill counts and dispensing records $^{11}{ }^{13}$ and is easily applied during medical visits. ${ }^{14}$ However, reliance on VAS also has its limitations. Patients tend to overestimate their level of adherence. ${ }^{11} 13$ Additionally, physicians have been found to be inaccurate in estimating patients' adherence when using VAS. ${ }^{15} 16$ These limitations may generate patient-physician discordance and impair the identification of patients with poor adherence. In turn, this might influence patient satisfaction and compromise shared decision making and therapeutic adjustments. ${ }^{17}$

Evidence is lacking on the degree and characteristics of discordance between patients and physicians in relation to the assessment of inhaled medication adherence. In other chronic diseases, patients' clinical status, disease severity and age are known predictors of patient-physician discordance regarding medication adherence. ${ }^{18} 19$ The identification of the level of discordance as well as characteristics associated with patient-physician discordance are essential to delineate effective strategies to maximise patient-physician agreement and improve clinical decisions. Therefore, we aimed to (1) compare patient's and physician's ratings of inhaled medication adherence using VAS and (2) to identify predictors of patientphysician discordance.

\section{METHODS}

\section{Study design}

Initial face-to-face visit and 1-week telephone interview data from two prospective observational studies of the Inspirers project were analysed (view online supplementary table 1). ${ }^{20}$ This project addresses the topic of adherence to asthma inhalers among adolescents and adults with persistent asthma. A convenience sample was recruited between November 2017 and June 2018 at 29 allergy, pulmonology and paediatric secondary care outpatient clinics in Portugal. The studies were approved by the ethics committees of all participating centers. The study is reported according to Strengthening the Reporting of Observational Studies in Epidemiology (STROBE) guidelines. ${ }^{21}$ Eligible patients were approached by physicians during medical visits. Adult patients signed a consent form. Adolescents signed an assent form, and a parental consent form was also obtained.

\section{Patients}

Patients were included if they had a previous medical diagnosis of persistent asthma, were at least 13 years old (13-17 years adolescents; $\geq 18$ years adults) and had an active prescription for an inhaled controller medication for asthma. All inhaled controller treatments were allowed, and there was no change in any prescribed medication in relation to the participation in these studies. Patients were excluded if they had a diagnosis of a chronic lung disease other than asthma or a diagnosis of another significant chronic condition with possible interference with the study aims.

\section{Patient and public involvement}

Patients were not involved in this study.

\section{Data collection}

During the initial face-to-face visit, data were collected from both patients and physicians. Physicians answered a questionnaire including: assessment of patients' asthma control according to the Global Initiative for Asthma $(\text { GINA })^{1}$; last known value of percent predicted forced expiratory volume in one second $\left(\mathrm{FEV}_{1}\right)$ and respective date; number of exacerbations, defined as episodes of progressive increase in shortness of breath, cough, wheezing and/or chest tightness, requiring change in maintenance therapy ${ }^{22}$; use of healthcare resources namely, number of unscheduled medical visits (primary 
care, secondary care or emergency department) and number of hospital admissions; and length of physicianpatient relationship. Physicians also reported on the patients' current asthma treatment, including inhaled and oral medication, allergen immunotherapy and biological therapy. Medication was grouped by active substance in classes: inhaled corticosteroids (ICS), long-acting betaagonists (LABA), ICS and LABA (ICS/LABA), long-acting muscarinic receptor antagonists (LAMA), short-acting beta-agonists (SABA), short-acting muscarinic-antagonists (SAMA), anti-leukotrienes, xanthines and oral corticosteroids. Reliever therapy with beta-agonist was classified based on the prescription of SABA and/or LABA and accounting for the type of prescribed LABA, considering that maintenance and reliever therapy (MART) is only recommended with formoterol. ${ }^{1}$ We stratified reliever therapy into three groups: without prescribed SABA or fast-acting LABA recommended for MART (includes patients prescribed a LABA other than formoterol); with SABA alone (also includes patients prescribed a LABA other than formoterol) and with LABA that allows MART (with or without concomitant SABA). In addition, we classified asthma severity in accordance with GINA treatment steps. ${ }^{1}$ Patients and physicians independently assessed patient global adherence to inhaled controller medication for asthma during the previous week using a VAS, ranging from 0 (worst) to 100 (best) $\mathrm{mm} .{ }^{23}$ Both filled in their respective VAS in distinct case report forms, without any specific instructions, and at distinct moments, being kept blind to each other's response.

Demographic data-age, gender, smoking habitswere also collected from patients during the face-toface visit. Upper airway control was assessed using the Control of Allergic Rhinitis and Asthma Test upper airway (CARAT-UA) subscore. ${ }^{24}$ The CARAT-UA subscore ranges from 0 to 12 points, with $>8$ points being indicative of good control. ${ }^{25}$

Approximately 1 week later, through a telephone interview, patients were asked to characterise the patientphysician relationship, namely if patient's preferences were considered at the time of inhaler prescription, if they had their inhaler technique reviewed during the last 12 months and if they had a written asthma action plan.

\section{Statistical analysis}

Descriptive statistics were used to characterise the sample. Normality of each variable was investigated with Kolmogorov-Smirnov tests and visual analysis of histograms. Inhaler adherence VAS scores were compared by pairing patients and physicians using Wilcoxon signed-rank tests and between adolescents and adults, using Mann-Whitney U tests, both considering patients and physicians scores. Intraclass correlation coefficient (ICC) and Bland-Altman plots were used to assess relative and absolute reliability of inhaler adherence VAS scores from patients and physicians. ICC was interpreted as excellent ( $\mathrm{ICC}>0.9$ ), good $(\mathrm{ICC}=0.75-0.9)$, moderate $(\mathrm{ICC}=0.5-0.75)$ or poor $($ ICC $<0.5) .{ }^{2627}$ Correlations with
Spearman's rho were also used to explore the relationship between patients and physicians VAS scores. Spearman's rho was interpreted as negligible $(0-0.30)$, low $(0.3-0.5)$, moderate $(0.5-0.7)$, high $(0.7-0.9)$ and very high $(0.9-1) .^{28}$

VAS scores were further categorised using cut-offs of $50 \%$ and $80 \%$, generating three VAS categories: low $(0-50 \mathrm{~mm})$, medium $(51-80 \mathrm{~mm})$ and high $(81-100 \mathrm{~mm})$ adherence. These cut-offs are frequently used for differentiation of adherence groups. ${ }^{29-33}$ To determine the agreement on VAS categories between patients and physicians, the percentage of agreement and weighted Cohen's kappa were used. ${ }^{34}$ Cohen's kappa values were interpreted as follows: $<0$, no agreement; $0-0.20$, slight; $0.21-0.40$, fair; $0.41-0.60$, moderate; $0.61-0.80$, substantial and $0.81-1.0$, almost perfect agreement. ${ }^{34}$

Discordance was defined as (1) category discordance, classification in distinct VAS categories or (2) value discordance, as an absolute difference in VAS scores $\geq 10 \mathrm{~mm}$. A difference of $10 \mathrm{~mm}$ has been widely accepted as a minimum threshold for clinical relevance. ${ }^{35-37}$ The direction of the discordance was characterised as physician overestimation of patient's adherence (higher VAS scores by at least $10 \mathrm{~mm}$ or higher VAS category) or, inversely, as physician underestimation (lower VAS scores by at least $10 \mathrm{~mm}$ or lower VAS category than the patients' perspective). Univariate multinomial logistic regressions were used to identify possible patient, disease or treatment characteristics predicting the discordance outcome $(0=$ concordance, used as reference; $1=$ physician underestimation; $2=$ physician overestimation). Variables with $\mathrm{p}<0.25$ at univariate analysis and informative variables linked to these variables (eg, last percent predicted $\mathrm{FEV}_{1}$ and respective date) were used in a multivariate multinomial logistic regression. ${ }^{38}$ When two candidate variables (predictors) were highly correlated, such as patient and physician adherence ratings, only one was included in the model ${ }^{39}$ The final model was obtained using a backward stepwise method of variable selection. Adjusted OR and $95 \%$ CI (OR (95\% CI) ) are presented. The overall model was evaluated using the goodness-of-fit tests and the Nagelkerke's R-square.

Statistical analyses were performed using IBM SPSS Statistics V.25.0 (IBM Corporation, Armonk, New York, USA) and plots were created using GraphPad Prism V.6.0 (GraphPad Software, La Jolla, California, USA). The level of significance was set at 0.05 .

\section{RESULTS}

\section{Participants}

From the 413 patients included in both studies, 395 (96\%) had complete data on inhaled medication adherence and were considered in this work. Patients had a median age (percentile 25 to percentile 75$)$ of 28 (16-46) years and were mainly female $(61 \%)$. Most were on ICS/LABA combination therapy $(\mathrm{n}=330 ; 84 \%)$ and used only one inhaler $(\mathrm{n}=265 ; 67 \%)$. According to the GINA classification, nearly 
Table 1 Participants' characteristics $(n=395)$

\begin{tabular}{|c|c|c|c|c|c|c|}
\hline \multicolumn{3}{|c|}{ Total $(n=395)$} & \multicolumn{2}{|c|}{ Adolescents ( $n=126)$} & \multicolumn{2}{|c|}{ Adults $(n=269)$} \\
\hline Age, median (P25-P75) & 28 & $(16-46)$ & 15 & $(14-16)$ & 40 & $(27-52)$ \\
\hline Female & 242 & (61) & 57 & (45) & 185 & (69) \\
\hline \multicolumn{7}{|l|}{ Smoking status } \\
\hline Never smoker & 301 & (76) & 115 & (91) & 186 & (69) \\
\hline Ex-smoker & 65 & (17) & 8 & (6) & 57 & (21) \\
\hline Current smoker & 27 & (7) & 3 & (2) & 24 & (9) \\
\hline \multicolumn{7}{|l|}{ Inhaled medication } \\
\hline ICS/LABA & 330 & (84) & 89 & (71) & 241 & (90) \\
\hline ICS & 66 & (17) & 37 & (29) & 29 & (11) \\
\hline LAMA & 50 & (13) & 3 & (2) & 47 & (18) \\
\hline LABA & 11 & (3) & 2 & (2) & 9 & (3) \\
\hline LABA/LAMA & 3 & (1) & 0 & & 3 & (1) \\
\hline SABA & 79 & (20) & 36 & (29) & 43 & (16) \\
\hline SAMA & 3 & (1) & 0 & & 3 & (1) \\
\hline \multicolumn{7}{|l|}{ Number of prescribed inhalers } \\
\hline 1 & 265 & (67) & 86 & (68) & 179 & (67) \\
\hline 2 & 113 & (29) & 40 & (32) & 73 & (27) \\
\hline$\geq 3$ & 16 & (4) & 0 & & 16 & (6) \\
\hline \multicolumn{7}{|l|}{ Oral medication } \\
\hline Anti-leukotrienes & 209 & (53) & 62 & (49) & 147 & (55) \\
\hline Xanthines & 12 & (3) & 0 & & 12 & (5) \\
\hline Oral corticosteroids & 8 & (2) & 0 & & 8 & (3) \\
\hline Allergen immunotherapy & 72 & (18) & 31 & (25) & 41 & (15) \\
\hline Biological therapy & 24 & (6) & 1 & (1) & 23 & (9) \\
\hline \multicolumn{7}{|l|}{ GINA assessment symptom control } \\
\hline Well-controlled & 209 & (53) & 58 & (46) & 151 & (56) \\
\hline Partly controlled/uncontrolled & 184 & (47) & 67 & (53) & 117 & (44) \\
\hline$\geq 1$ exacerbations past year & 195 & (49) & 70 & (56) & 125 & (47) \\
\hline $\begin{array}{l}\geq 1 \text { unscheduled medical visits past } \\
\text { year }\end{array}$ & 120 & (30) & 38 & (30) & 82 & (31) \\
\hline$\geq 1$ hospital admissions past year & 15 & (4) & 2 & (2) & 13 & (5) \\
\hline
\end{tabular}

Values are shown as $\mathrm{n}(\%)$ unless otherwise indicated.

GINA, Global Initiative for Asthma; ICS, inhaled corticosteroids; LABA, long-acting beta-agonists; LAMA, long-acting muscarinic receptor antagonists;P25-P75, percentile 25 to percentile 75; SABA, short-acting beta-agonists; SAMA, short-acting muscarinic-antagonists.

half of participants had their asthma not well-controlled $(\mathrm{n}=184 ; 47 \%)$ and had at least one exacerbation during the previous year $(\mathrm{n}=195 ; 49 \%)$. Characteristics of the participants are summarised in table 1.

\section{Inhaler adherence-patient reported and physician assessment}

Inhaler adherence was considered high both by patients (median 85 (65-95) mm, VAS $>8053 \%$ ) and by physicians (84 (68-95) mm, VAS $>8053 \%)$. VAS scores were significantly lower in adolescents when compared with adults, in the perspective of both patients (median 80 vs 88 , $\mathrm{p}<0.001$ ) and physicians (median 79 vs $88, \mathrm{p}<0.001$ ). The median difference between patients and physicians VAS scores was significantly higher for adolescents than adults (median $11(5-20)$ vs 9 (3-20); $\mathrm{p}=0.025)$. Correlation between patient and physician VAS scores was moderate $\left(\mathrm{r}_{\mathrm{s}}=0.580 ; \mathrm{p}<0.001\right) \quad$ (figure 1$)$. A lower correlation was found for adolescents $\left(r_{s}=0.462\right.$ vs adults $\left.r_{s}=0.572\right)$. The relative reliability between patients and physicians scores was moderate, with an ICC of 0.63 (95\% CI 0.57 to 0.69 ). Reliability in adolescents was 0.52 (0.38 to 0.64$)$ and in adults 0.66 (0.59 to 0.73 ). Bland and Altman plots are shown in figure 2 . There was reasonable agreement, with bias close to zero and quite large limits of agreement 


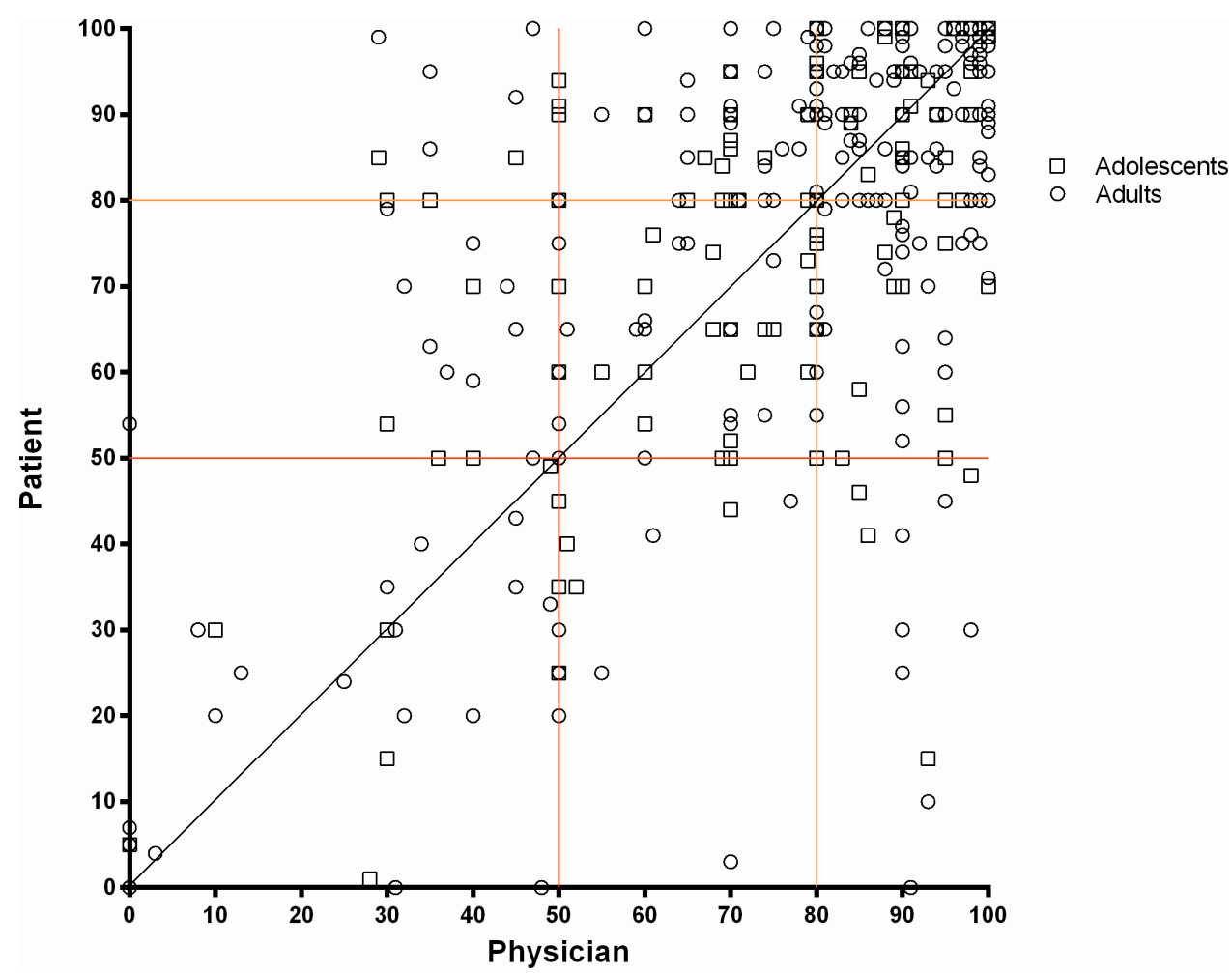

Figure 1 Scatter plot showing the relationship between patients and physicians estimates of inhaler adherence $(n=395)$, with the black line representing perfect agreement; the red and orange lines representing the cut-offs of 50 and $80-$ in $40 \%$ cases both patients and physicians classified adherence to inhaler treatments in previous week higher than $80 \%$, in $15 \%$ cases between $51 \%$ and $80 \%$ and in $9 \%$ cases below 50\%. Physicians underestimated adherence in $19 \%$ and overestimated adherence in $17 \%$ of the participants.

(LoA) (bias 0.15, SD 20.5; 95\% LoA -40.1-40.4). Considering the two age groups, a slightly better agreement for adults (bias 0.52 , SD 20.3; 95\% LoA -39.3-40.4) in comparison with adolescents (bias -0.64, SD 20.9; $95 \%$ LoA -41.7-40.4) was found.

Value discordance was high $(\mathrm{n}=211 ; 53 \%)$, with physicians overestimating adherence in $26 \%(\mathrm{n}=102)$ of cases and underestimating it in $27 \%(\mathrm{n}=109)$. Category discordance occurred in $36 \%(\mathrm{n}=142)$ of cases, with physicians overestimating adherence in $17 \% \quad(n=66)$ of cases and underestimating in $19 \%(\mathrm{n}=76)$ (table 2$)$. Based on category discordance, a weighted Cohen's kappa of $0.46(0.38$ to 0.54$)(\mathrm{p}<0.001)$ was found, reflecting moderate agreement. The category discordance between patients and physicians was higher in adolescents $(41 \%)$ than in adults $(34 \%)$. Weighted kappa was $0.36(0.22$ to 0.50$)$ for adolescents and 0.48 (0.39 to 0.58$)$ for adults, demonstrating a fair and moderate agreement, respectively.

Total discordance occurred in 56\% of cases: 78 (20\%) based on value discordance only, $9(2 \%)$ based on category discordance only and $133(34 \%)$ classified as discordant using both methods. In $28 \%$ of the cases, physicians overestimated adherence and in $27 \%$ underestimated it. Unadjusted ORs estimating the association between each variable and direction of physician discordance are presented in online supplementary table 2 . Adjusted ORs of the multivariate multinomial model are summarised in table 3. Uncontrolled asthma (OR 2.33 (1.25 to 4.34)),
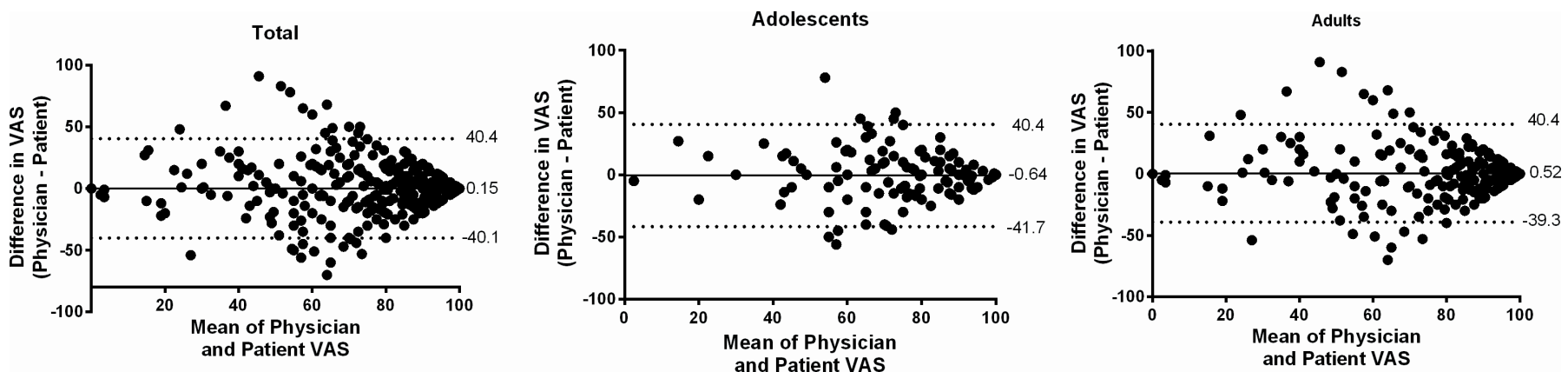

Figure 2 Bland-Altman plots of inhaler adherence Visual Analogue Scale (VAS) scores between patients and physicians in the total sample $(n=395)$, in adolescents $(n=126)$ and in adults $(n=269)$. The solid lines represent the bias, and the dashed lines show the $95 \%$ limits of agreement. 
Table 2 Agreement on VAS categories between patients and physicians ( $n=395)-64 \%$ were in the same category, 30\% differed one category and $6 \%$ differed two categories

\begin{tabular}{|c|c|c|c|c|c|c|c|c|c|}
\hline \multirow{4}{*}{$\begin{array}{l} \\
\text { Physician } \\
\text { VAS category }\end{array}$} & \multirow[b]{3}{*}{ High } & \multicolumn{8}{|c|}{ Patient VAS category } \\
\hline & & \multicolumn{2}{|l|}{ High } & \multicolumn{2}{|c|}{ Medium } & \multicolumn{2}{|c|}{ Low } & \multicolumn{2}{|c|}{ Total } \\
\hline & & 156 & $(40 \%)$ & 41 & (10\%) & 13 & (3\%) & 210 & (53\%) \\
\hline & Medium & 43 & $(11 \%)$ & 61 & $(15 \%)$ & 12 & $(3 \%)$ & 116 & $(29 \%)$ \\
\hline & Low & 10 & $(3 \%)$ & 23 & $(6 \%)$ & 36 & $(9 \%)$ & 69 & $(18 \%)$ \\
\hline & Total & 209 & (53\%) & 125 & (32\%) & 61 & (15\%) & 395 & (100\%) \\
\hline
\end{tabular}

Values in bold represent perfect agreement.

VAS, Visual Analogue Scale.

uncontrolled upper airway disease (OR 2.86 (1.35 to $6.04)$ ) and prescription of SABA alone (OR 3.05 (1.15 to 8.08)) were predictors of overestimation. Low adherence rated by the physician (OR 27.35 (9.85 to 75.95)), $\mathrm{FEV}_{1} \geq 80 \%$ (OR 2.59 (1.08 to 6.20)) and a first appointment (OR 5.63 (1.24 to 25.56)) predicted underestimation. Medium adherence as assessed by the physician was significantly associated with higher risk of both physician overestimation and underestimation (OR 14.50 (6.04 to 34.81 ) and OR 2.21 (1.07 to 4.58), respectively), while having a written action plan decreased the likelihood of discordance (OR 0.25 (0.12 to 0.52 ) and OR 0.41 (0.22 to $0.78)$, respectively). Pearson $\chi^{2}(\mathrm{p}=0.431)$ and deviance $(\mathrm{p}=0.721)$ showed that the multinomial logistic regression model adequately fitted the data. This model explained $44 \%$ of the variance in patient-physician discordance and correctly classified $63 \%$ of cases.

\section{DISCUSSION}

To the authors' knowledge, this is the first study investigating patient and physician agreement on adherence to

Table 3 Multivariate multinomial model to explain physician overestimation or underestimation of patient's adherence (patient-physician concordance used as reference, $n=142,45 \%$ )

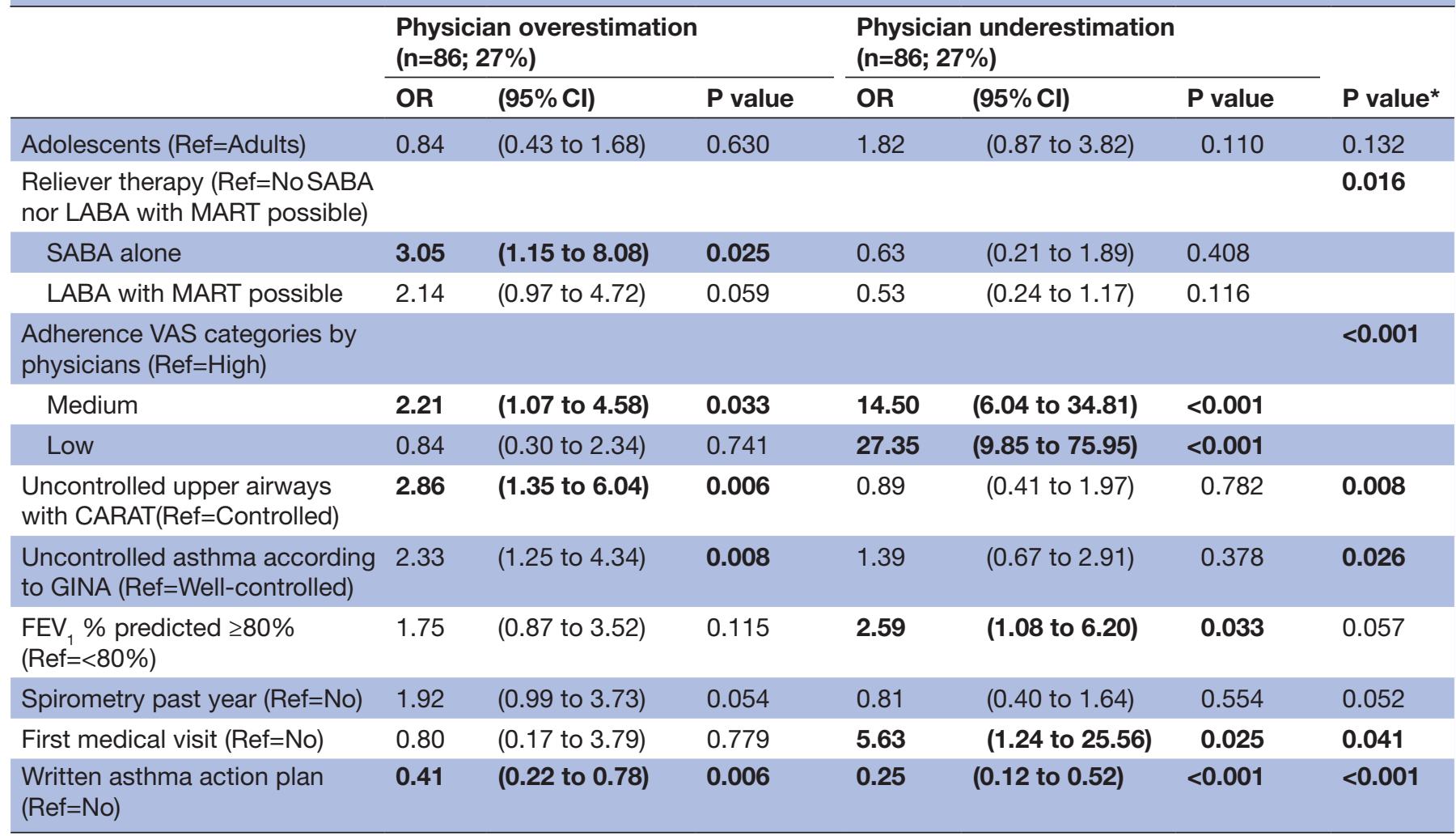

314 patients included in this analysis as clinical information was incomplete for 81 patients, mainly lacking information on FEV $_{1}$. Significant values marked in bold.

*Likelihood ratio tests.

CARAT, Control of Allergic Rhinitis and Asthma Test; FEV , forced expiratory volume in one second; GINA, Global Initiative for Asthma; LABA, long-acting beta-agonists; MART, maintenance and reliever therapy; SABA, short-acting beta-agonists; VAS, Visual Analogue Scale. 
asthma inhalers. We found that although both patients and physicians reported high inhaler adherence, their degree of adherence was discordant in half of the cases. We have also identified in a multinomial model the predictors of this discordance. Uncontrolled asthma, uncontrolled upper airway disease and prescription of SABA alone were predictors of physician's overestimation of adherence, while low adherence as assessed by the physician (VAS $\leq 50 \mathrm{~mm}$ ), $\mathrm{FEV}_{1}$ $\geq 80 \%$ and being on a first appointment were predictors of underestimation. Medium inhaler adherence rated by the physician (VAS 51-80 $\mathrm{mm}$ ) and absence of a written action plan were predictors of both physician overestimation and underestimation.

Self-reported adherence to inhaled medications was found to be high (median 85), which is in line with findings from previous studies on adherence to inhaled medication (mean 90) ${ }^{32} 33$ and to oral medications (mean or median values between 84 and 100). ${ }^{11} 131940-44$ Physicians' perception of the extent of adherence to inhaled medication was also high (median $84 \mathrm{~mm}$ ), in agreement with prior reports. ${ }^{14} 1842-44$ Adherence self-reported by adolescents and assessed by their physicians was lower in comparison with adults. This was somewhat expected as previous research showed that adolescents tend to be less adherent to inhaled asthma therapy when compared with younger children or adults. ${ }^{45}{ }^{46}$ Irrespective of the patients' age, it is possible that both patients' and physicians' adherence estimates were overestimating real adherence, as previous research showed lower adherence levels to inhaled medications for asthma when using objective assessment methods ${ }^{4}$ and overestimation of subjective measures of adherence when compared with objective measures. ${ }^{15} 18$ 47-49 However, in the present study, we did not collect objective data on inhaler adherence to support this assumption. ${ }^{47}$ This was mainly due to the fact that currently available objective methods (eg, medical/dispensing records, electronic monitoring devices) required laborious analysis by physicians and were costly to implement in clinical practice. There is an urgent need to develop and validate low-cost, ubiquitous and easy-to-disseminate tools to objectively measure inhaler adherence. Mobile applications that allow patients to record their inhaler adherence and share it with their physician may be promising solutions. Some apps with these features are already available, such as AsthmaMD (https://www.asthmamd.org/), Asthma Coach (http://myhealthapps.net/app/details/317/ asthma-coach) and InspirerMundi (https://play.google. $\mathrm{com} /$ store/apps/details?id=com.bloomidea.inspirers\& hl=pt_PT). InspirerMundi app, besides traditional daily self-report of medication intake, has an additional inhaled medication adherence detection tool, using the smartphone camera and advanced image processing, which validates inhaler use through dose tracking. ${ }^{50}$ The Inspirers research project, in which the present work is included, is developing the InspirerMundi app and plans to assess its feasibility and validity in real-life studies.

Discordance was found in 36\% (VAS categories) and 53\% of cases (VAS difference $\geq 10 \mathrm{~mm}$ ) and fair-to-moderate agreement was found between patient and physician reports. In other conditions requiring daily therapy, such as HIV, similar discordance rates have been found. ${ }^{1851}$ This discordant perspective regarding medication adherence is of particular concern as it may limit evaluation and discussion of treatment decisions between patients and their physicians (shared decision making), ${ }^{17}$ which is likely to have a negative impact on diagnostic and therapeutic decisions such as unnecessary additional procedures and adjustments to medication regimen. In future, implementation of more effective patient-physician communication together with the use of objective adherence measures at the time of the medical encounter may improve agreement.

Irrespective of the method used to define discordance, we found a balance between physicians overestimation and underestimation of inhaler adherence compared with patient self-reported adherence. In studies conducted in other chronic diseases, such as hypertension, osteoporosis or HIV, physicians tended to overestimate adherence. ${ }^{151847}$ One can speculate that this compared overestimation and underestimation may be related to a higher awareness of the barriers to inhaled medication adherence of physicians treating patients with asthma. However, studies with objective adherence measures evaluating the predictors for physicians underestimation and overestimation of adherence are needed.

The discordance in the perceptions of inhaler adherence may have several reasons. In this study, having a written asthma action plan was associated with a reduction in the likelihood of discordance. If patients and physicians at a certain point of their relationship have agreed on an action plan, they will be more likely to implement effective communication, partnership working and shared decision making during medical visits, which is also reflected in closer estimates of inhaler adherence. Indeed, effective communication was one of the main themes identified by patients as helping the promotion and/or use of action plans. ${ }^{52}$ In the patients' view, good communication meant being listened to, feeling respected and having their own knowledge and experience of asthma recognised by physicians during clinical encounters. ${ }^{52}$ The same argument may also explain why absence of previous patient-physician relationship was associated with underestimation of adherence. Implementation of effective communication in the first visit is challenging, as it is a critical time for a systematic collection of diverse information. ${ }^{53}$ First visits were also previously linked to greater discordance between patients and physicians. ${ }^{183}$

Other predictors of overestimation were prescription of SABA alone, uncontrolled asthma and uncontrolled upper airway disease. Although in this study we have not quantified the use of reliever therapy, the inclusion of SABA alone in the physician-reported therapeutic plan can be regarded as a proxy of SABA use. These associations suggest that physicians consider patients with poor outcomes or using SABA as being more likely to adhere to the prescribed control inhaler. However, this is contrary to the available 
evidence showing that SABA can adversely affect adherence to anti-inflammatory treatment and contribute to high levels of poorly controlled asthma. ${ }^{54}$ Thus, we cannot exclude a possible reverse causality with overestimation itself being a marker of underlying lack of effective patientphysician communication, which might lead to impaired physician perception of the need for control medication adjustments and contribute to worse patient outcomes, including poor disease control and need for SABA. Nevertheless, the estimation of adherence in light of disease control might be highly misleading, as patients could have their disease controlled even with dosages much lower than prescribed, whereas others who take their prescribed medication may not. ${ }^{19}$ Indeed, some studies demonstrate that asthma control is not directly associated with adherence behaviours. ${ }^{556}$ The same argument may also explain why an $\mathrm{FEV}_{1} \geq 80 \%$, also related to good asthma control, ${ }^{5758}$ was associated with physician underestimation of adherence, suggesting that physicians believe that patients who have better lung function are more prone to forget or reduce medication intake. It is known that, in the absence of objective adherence data, physicians commonly rely on patients' health status to estimate adherence. This is not exclusive of physicians managing patients with asthma and occurs also in other diseases. ${ }^{1519}$ Nevertheless, this is a dangerous assumption that may lead to unnecessary and inadequate changes in the medication regimen.

Our study has some limitations. Although data come from two multicentre studies involving 29 secondary care outpatient clinics, patients were recruited by convenience sampling. Therefore, the findings may not be generalisable to all patients with persistent asthma. Future studies can confirm or negate these results, preferably recruiting patients from different healthcare settings, including primary care, and using consecutive or random sampling. Patient and physician estimates were assessed only at one time-point and based on a single type of measure (VAS). Future long-term studies could use VAS together with other adherence subjective measures, such as the Eight-Item Morisky Medication Adherence Scale, ${ }^{59}$ and with complementary questionnaires on adherence issues such as medication beliefs ${ }^{60}$ or knowledge. ${ }^{61}$ Also, future research could compare subjective estimates with objective adherence measures, ideally simple to use during clinical encounters. When interpreting the agreement between patients and physicians, we need to be aware that kappa is highly sensitive to the distribution of the marginal totals and could produce unreliable results. Also, we need to take into account that each physician rated several patients and a possible physician-cluster effect might have occurred. In addition, as there is no standardised way to define discordance, we used two approaches to define discordance, both based on previously used and generally accepted cut-offs, ${ }^{29-31}{ }^{35-37}$ which we believe make our results more robust. Nevertheless, more research is needed on how to define discordance. Regarding discordance prediction, some outcomes of interest occurred frequently $(>10 \%)$ and this may lead to overestimation of the relative risk. This problem has been identified in cohort studies of common outcomes, ${ }^{62}$ and thus our results need to be interpreted with caution.

In conclusion, although both patients and physicians report high inhaler adherence, discordance occurs in half of cases. This study has identified some predictors that can help to improve the understanding on this discordance. Implementation of objective adherence measures and effective communication are needed to improve the patient-physician agreement and therapeutic decisions.

\section{Author affiliations}

${ }^{1}$ Center for Health Technology and Services Research (CINTESIS), Faculty of Medicine, University of Porto (FMUP), Porto, Portugal

${ }^{2}$ Immunoalergology, CUF-Porto Hospital and Institute, Porto, Portugal

${ }^{3}$ Serviço de Pediatria, Centro Materno Infantil do Norte, Centro Hospitalar Universitário do Porto, Porto, Portugal

${ }^{4}$ MEDIDA - Medicina, Educação, Investigação, Desenvolvimento e Avaliação, Porto, Portugal

${ }^{5}$ Serviço de Pneumologia A, Hospital Universitário de Coimbra, Centro Hospitalar e Universitário de Coimbra EPE, Coimbra, Portugal

${ }^{6}$ Unidade de Imunoalergologia, Unidade Local de Saúde de Matosinhos EPE, Senhora da Hora, Portugal

${ }^{7}$ Imunologia Básica e Clínica, Faculdade de Medicina, Universidade do Porto, Porto, Portugal

${ }^{8}$ Serviço de Imunoalergologia, Hospital de Santa Maria, Centro Hospitalar Lisboa Norte EPE, Lisboa, Portugal

${ }^{9}$ Serviço de Pediatria, Unidade Local de Saúde de Matosinhos EPE, Senhora da Hora, Portugal

${ }^{10}$ Serviço de Pneumologia B, Hospital Geral, Centro Hospitalar e Universitário de Coimbra EPE, Coimbra, Portugal

${ }^{11}$ Serviço de Imunoalergologia, Hospital Universitário de Coimbra, Centro Hospitalar e Universitário de Coimbra EPE, Coimbra, Portugal

${ }^{12}$ Serviço de Imunoalergologia, Hospital de Dona Estefânia, Centro Hospitalar de Lisboa Central EPE, Lisboa, Portugal

${ }^{13}$ Pathophysiology, CEDOC, Integrated Pathophysiological Mechanisms Research Group, Nova Medical School, Lisboa, Portugal

${ }^{14}$ Serviço de Pneumologia, Hospital Beatriz Ângelo, Loures, Portugal

${ }^{15}$ Serviço de Pediatria, Hospital da Senhora da Oliveira, Guimaraes, Portugal

${ }^{16}$ Serviço de Imunoalergologia, Hospital Amato Lusitano, Castelo Branco, Portugal

${ }^{17}$ Serviço de Imunoalergologia, Centro Hospitalar do Algarve EPE, Faro, Portugal

${ }^{18}$ Serviço de Imunoalergologia, Hospital São João, Porto, Portugal

${ }^{19}$ University of Beira Interior, CICS - Health Sciences Research Centre; NuESA Environment \& Health Study Group, Faculty of Health Sciences, Covilha, Portugal

${ }^{20}$ Cova da Beira University Hospital Centre, Department of Allergy \& Clinical

Immunology, Covilhã, Portugal

${ }^{21}$ Unidade de Imunoalergologia, Hospital do Divino Espirito Santo de Ponta Delgada EPE, Ponta Delgada, Portugal

${ }^{22}$ Serviço de Pneumologia, Centro Hospitalar de Trás-os-montes e Alto Douro EPE,

Vila Real, Portugal

${ }^{23}$ Imunoalergologia, Grupo HPA Saúde, Portimao, Portugal

${ }^{24}$ Serviço de Pneumologia, Centro Hospitalar Barreiro Montijo EPE, Barreiro, Portugal

${ }^{25}$ Serviço de Pediatria, Centro Hospitalar do Médio Ave EPE, Santo Tirso, Portugal

${ }^{26}$ Pediatrics, Hospital de Santa Maria, Lisbon, Portugal

${ }^{27}$ Laboratory of Clinical Pharmacology and Therapeutics, University of Lisbon

Medical Faculty, Lisboa, Portugal

${ }^{28}$ Serviço de Pneumologia, Hospital Garcia de Orta EPE, Almada, Portugal

${ }^{29}$ Serviço de Pediatria, Centro Hospitalar Tondela Viseu EPE, Viseu, Portugal

${ }^{30}$ Pneumology, Centro Hospitalar de Lisboa Central, Lisbon, Portugal

${ }^{31}$ NOVA Medical School, Lisbon, Portugal

${ }^{32}$ Imunoalergologia, Centro de Imunoalergologia do Algarve, Portimão, Portugal

${ }^{33}$ Serviço de Imunoalergologia, Serviço de Saúde da Região Autónoma da Madeira, Funchal, Portugal

${ }^{34}$ Serviço de Imunoalergologia, Centro Hospitalar Vila Nova de Gaia/Espinho, Vila Nova de Gaia, Portugal

${ }^{35}$ Bloco operatório, Centro Hospitalar Vila Nova de Gaia/Espinho, Vila Nova de Gaia, Portugal 
${ }^{36}$ Department of Community Medicine, Information and Health Decision Sciences (MEDCIDS), University of Porto Faculty of Medicine, Porto, Portugal

Acknowledgements We thank the participants and centers involved in the project Inspirers. We thank Adelaide Alves, Ana Castro Neves, Anabela Lopes, Andreia Oliveira, Ângelo Afonso, Catarina Parra, Cátia Alves, David Trincão, Diana Pinto, Elena Finelli, Elisa Pedro, Fernanda Carvalho, Filipa Lopes, Joana Borges, Joana Branco, José Ferraz de Oliveira, Liliana Ribeiro, Margarida Valério, Mariana Conde, Marta Pereira, Sara Cabral, Sara Prates and Teresa Almeida for their support in data collection. We thank Rita Amaral for her support in the statistical analyses.

Collaborators Inspirers group.

Contributors CJ, AMP, RA, MF-M, MACorreia, CCL, AM, AMA, ATB, JA, PLP, JGM, AGC, LT-B, MFT, RF, JAlberto Ferreira, AC and JAlmeida Fonseca helped design the study. CJ and AMP contributed to statistical analysis. CJ drafted the manuscript. CJ, AMP, RA, MF-M, MC, LA, MP, MACorreia, CCL, MJoana Catarata, LMS, JP, BR, CLopes, AMendes, JCCR, GO, APA, IA, JCarvalho, AMA, JCC, JD, ATB, JA, CR, MA, PLP, NN, AP, JGM, NP, PM, FTB, MAS, AGC, ASN, MS, CLozoya, NS, DS, MJV, LT-B, CC, MFT, RRA, ASM, CSP, PMS, CA, RCâmara, DC, DB, RMF, RF, FM, RGomes, MJosé Calix, AMarques, JCardoso, ME, RGerardo, CN, RCâmara, JAlberto Ferreira, AC, PF RCorreia and JAlmeida Fonseca contributed to data collection, interpretation and discussion of the results and approval of the final version of the manuscript.

Funding $C J$ is a post-doc fellow (SFRH/BPD/115169/2016) funded by Fundação para a Ciência e Tecnologia (FCT), reimbursed by Fundo Social Europeu and by national funds of MCTES. This work was funded by ERDF (European Regional Development Fund) through the operations: P0Cl-01-0145-FEDER-029130 ('mINSPIRERS - mHealth to measure and improve adherence to medication in chronic obstructive respiratory diseases-generalisation and evaluation of gamification, peer support and advanced image processing technologies') cofunded by the COMPETE2020 (Programa Operacional Competitividade e Internacionalização), Portugal 2020 and by Portuguese Funds through FCT (Fundação para a Ciência e a Tecnologia)

Competing interests None declared.

Patient consent for publication Not required.

Ethics approval The studies were approved by distinct ethics committees (CUF Porto Instituto \& Hospital, Centro Hospitalar e Universitário de Coimbra, Unidade Local de Saúde de Matosinhos, Centro Hospitalar Lisboa Norte, Centro Hospitalar Universitário de Lisboa Central, Hospital Beatriz Ângelo, Hospital da Senhora da Oliveira, Unidade Local de Saúde de Castelo Branco, Centro Hospitalar Universitário do Algarve, Centro Hospitalar de São João, Centro Hospitalar Universitário Cova da Beira, Centro Hospitalar Universitário do Porto, Centro Hospitalar de Trás-os-Montes e Alto Douro, Centro Hospitalar Barreiro Montijo, Centro Hospitalar do Médio Ave, Hospital Garcia de Orta, Centro Hospitalar Tondela-Viseu, Serviço de Saúde da Região Autónoma da Madeira, Centro Hospitalar Vila Nova de Gaia/Espinho, Hospital Privado de Alfena) and by the National Data Protection Committee (no 8825/ 2017 and no 11060/ 2017).

Provenance and peer review Not commissioned; externally peer reviewed.

Data availability statement The data sets generated during and/or analysed during the current study are not publicly available.

Open access This is an open access article distributed in accordance with the Creative Commons Attribution Non Commercial (CC BY-NC 4.0) license, which permits others to distribute, remix, adapt, build upon this work non-commercially, and license their derivative works on different terms, provided the original work is properly cited, appropriate credit is given, any changes made indicated, and the use is non-commercial. See: http://creativecommons.org/licenses/by-nc/4.0/.

ORCID iDs

Cristina Jácome http://orcid.org/0000-0002-1151-8791

Rute Almeida http://orcid.org/0000-0001-7755-5002

Luís Taborda-Barata http://orcid.org/0000-0001-6649-8890

\section{REFERENCES}

1 Global Initiative for Asthma. Global strategy for asthma management and prevention, 2018. Available: www.ginasthma.org

2 O'Byrne PM, Barnes PJ, Rodriguez-Roisin R, et al. Low dose inhaled budesonide and formoterol in mild persistent asthma: the optima randomized trial. Am J Respir Crit Care Med 2001;164:1392-7.
3 Pauwels RA, Pedersen S, Busse WW, et al. Early intervention with budesonide in mild persistent asthma: a randomised, double-blind trial. The Lancet 2003;361:1071-6.

4 Engelkes M, Janssens HM, de Jongste JC, et al. Medication adherence and the risk of severe asthma exacerbations: a systematic review. Eur Respir J 2015;45:396-407.

5 Stanford RH, Averell C, Parker ED, et al. Assessment of adherence and asthma medication ratio for a once-daily and twice-daily inhaled Corticosteroid/Long-Acting $\beta$-Agonist for asthma. J Allergy Clin Immunol 2019;7:1488-96.

6 Doz M, Chouaid C, Com-Ruelle L, et al. The association between asthma control, health care costs, and quality of life in France and Spain. BMC Pulm Med 2013;13:15.

7 Bender B, Milgrom H, Apter A. Adherence intervention research: what have we learned and what do we do next? J Allergy Clin Immunol 2003;112:489-94.

8 Lima-Dellamora EdaC, Osorio-de-Castro CGS, Madruga LGDSL, et al. Use of pharmacy records to measure treatment adherence: a critical review of the literature. Cad Saude Publica 2017;33:e00136216.

9 van Heuckelum M, van den Ende CHM, Houterman AEJ, et al. The effect of electronic monitoring feedback on medication adherence and clinical outcomes: a systematic review. PLoS One 2017; 12:e0185453

10 Garfield S, Clifford S, Eliasson L, et al. Suitability of measures of selfreported medication adherence for routine clinical use: a systematic review. BMC Med Res Methodol 2011;11:149.

11 Kalichman SC, Amaral CM, Swetzes C, et al. A simple single-item rating scale to measure medication adherence: further evidence for convergent validity. J Int Assoc Physicians AIDS Care 2009;8:367-74.

12 Lam WY, Fresco P. Medication adherence measures: an overview. Biomed Res Int 2015;2015:12

13 Nau DP, Steinke DT, Williams LK, et al. Adherence analysis using visual analog scale versus claims-based estimation. Annals of Pharmacotherapy 2007;41:1792-7.

14 Klok T, Kaptein AA, Duiverman EJ, et al. Long-Term adherence to inhaled corticosteroids in children with asthma: observational study. Respir Med 2015;109:1114-9.

15 Zeller A, Taegtmeyer A, Martina B, et al. Physicians' ability to predict patients' adherence to antihypertensive medication in primary care. Hypertens Res 2008;31:1765-71.

16 Gebbia V, Bellavia M, Banna GL, et al. Treatment monitoring program for implementation of adherence to second-line erlotinib for advanced Non-Small-Cell lung cancer. Clin Lung Cancer 2013;14:390-8

17 Liew SM, Lee WK, Khoo EM, et al. Can doctors and patients correctly estimate cardiovascular risk? A cross-sectional study in primary care. BMJ Open 2018;8:e017711.

18 Miller LG, Liu H, Hays RD, et al. How well do clinicians estimate patients' adherence to combination antiretroviral therapy? J Gen Intern Med 2002;17:1-11.

19 Velligan DI, Wang M, Diamond P, et al. Relationships among subjective and objective measures of adherence to oral antipsychotic medications. PS 2007:58:1187-92.

20 Jácome C, Guedes R, Almeida R, et al. mINSPIRERS - Estudo da exequibilidade de uma aplicação móvel para medição e melhoria da adesão medicação inalada de controlo em adolescentes e adultos com asma persistente. Protocolo de um estudo observacional multicêntrico. Revista Portuguesa de Imunoalergologia 2018;26:47-61.

21 Vandenbroucke JP, von Elm E, Altman DG, et al. Strengthening the reporting of observational studies in epidemiology (STROBE): explanation and elaboration. Int J Surg 2014;12:1500-24.

22 Loymans RJB, ter Riet G, Sterk PJ. Definitions of asthma exacerbations. Curr Opin Allergy Clin Immunol 2011;11:181-6.

23 Jonsdottir H, Opjordsmoen S, Birkenaes AB, et al. Medication adherence in outpatients with severe mental disorders: relation between self-reports and serum level. J Clin Psychopharmacol 2010;30:169-75

24 Fonseca JA, Nogueira-Silva L, Morais-Almeida M, et al. Validation of a questionnaire (CARAT10) to assess rhinitis and asthma in patients with asthma. Allergy 2010;65:1042-8.

25 Fonseca JA, Nogueira-Silva L, Morais-Almeida M, et al. Control of allergic rhinitis and asthma test (CARAT) can be used to assess individual patients over time. Clin Trans/ Allergy 2012;2:16

26 Fleiss J. Reliability of measurement. In: Fleiss J, ed. Design and analysis of clinical experiments. New York: John Wiley \& Sons, 1986: 1-32.

27 Koo TK, Li MY. A guideline of selecting and reporting intraclass correlation coefficients for reliability research. J Chiropr Med 2016:15:155-63. 
28 Mukaka MM. Statistics corner: a guide to appropriate use of correlation coefficient in medical research. Malawi Med $J$ 2012;24:69-71.

29 van Boven JFM, Chavannes $\mathrm{NH}$, van der Molen T, et al. Clinical and economic impact of non-adherence in COPD: a systematic review. Respir Med 2014;108:103-13.

30 Humenberger M, Horner A, Labek A, et al. Adherence to inhaled therapy and its impact on chronic obstructive pulmonary disease (COPD). BMC Pulm Med 2018;18:163.

31 Yu S, Burge RT, Foster SA, et al. The impact of teriparatide adherence and persistence on fracture outcomes. Osteoporos Int 2012;23:1103-13.

32 Bozek A, Jarzab J. Adherence to asthma therapy in elderly patients. Journal of Asthma 2010;47:162-5.

33 Bae Y-J, Kim T-B, Jee Y-K, et al. Severe asthma patients in Korea overestimate their adherence to inhaled corticosteroids. Journal of Asthma 2009;46:591-5.

34 Landis JR, Koch GG. The measurement of observer agreement for categorical data. Biometrics 1977;33:159-74.

35 Lal SD, McDonagh J, Baildam E, et al. Agreement between proxy and adolescent assessment of disability, pain, and well-being in juvenile idiopathic arthritis. J Pediatr 2011;158:307-12.

36 Steinsbekk A, Lüdtke R. Patients' assessments of the effectiveness of homeopathic care in Norway: A prospective observational multicentre outcome study. Homeopathy 2005:94:10-16.

37 Johnson MJ, Bland JM, Oxberry SG, et al. Clinically important differences in the intensity of chronic refractory breathlessness. $J$ Pain Symptom Manage 2013;46:957-63.

38 Hosmer DW, Lemeshow S, Sturdivant RX. Model-Building Strategies and Methods for Logistic Regression. In: Hosmer J DW, Lemeshow $\mathrm{S}$, Sturdivant RX, eds. Applied logistic regression. 3rd edn. New Jersey: John Wiley \& Sons, Inc, 2013.

39 Ranganathan P, Pramesh CS, Aggarwal R. Common pitfalls in statistical analysis: logistic regression. Perspect Clin Res 2017;8:148-51.

40 Wohl DA, Panter AT, Kirby C, et al. Estimating HIV medication adherence and persistence: two instruments for clinical and research use. AIDS Behav 2018;22:948-60.

41 Amico KR, Fisher WA, Cornman DH, et al. Visual analog scale of art adherence: association with 3-day self-report and adherence barriers. J Acquir Immune Defic Syndr 2006;42:455-9.

42 Feldman DE, Civita Mde, Dobkin PL, et al. Perceived adherence to prescribed treatment in juvenile idiopathic arthritis over a one-year period. Arthritis Rheum 2007;57:226-33.

43 Walsh JC, Horne R, Dalton M, et al. Reasons for non-adherence to antiretroviral therapy: patients' perspectives provide evidence of multiple causes. AIDS Care 2001;13:709-20.

44 Mazzeo F, Duck L, Joosens E, et al. Nonadherence to imatinib treatment in patients with gastrointestinal stromal tumors: the ADAGIO study. Anticancer Res 2011;31:1407-9.

45 Dima AL, Hernandez G, Cunillera O, et al. Asthma inhaler adherence determinants in adults: systematic review of observational data. Eur Respir J 2015;45:994-1018.
46 Koster ES, Raaijmakers JAM, Vijverberg SJH, et al. Inhaled corticosteroid adherence in paediatric patients: the PACMAN cohort study. Pharmacoepidemiol Drug Saf 2011;20:1064-72.

47 Curtis JR, Cai Q, Wade SW, et al. Osteoporosis medication adherence: physician perceptions vs. patients' utilization. Bone 2013;55:1-6.

48 Liu H, Golin CE, Miller LG, et al. A comparison study of multiple measures of adherence to HIV protease inhibitors. Ann Intern Med 2001;134:968-77.

49 Goldstein TR, Krantz M, Merranko J, et al. Medication adherence among adolescents with bipolar disorder. J Child Adolesc Psychopharmacol 2016;26:864-72.

50 Jácome C, Almeida R, Teixeira J, et al. Inspirers: an APP to measure and improve adherence to inhaled treatment. Lisbon: International Conference e-Health; 2017.

51 Murri R, Ammassari A, Trotta MP, et al. Patient-Reported and physician-estimated adherence to HAART: social and clinic centerrelated factors are associated with discordance. J Gen Intern Med 2004;19:1104-10.

52 Ring N, Jepson R, Hoskins G, et al. Understanding what helps or hinders asthma action plan use: a systematic review and synthesis of the qualitative literature. Patient Educ Couns 2011;85:e131-43.

53 Coran JJ, Koropeckyj-Cox T, Arnold CL. Are physicians and patients in agreement? exploring Dyadic concordance. Health Educ Behav 2013;40:603-11.

54 Martin MJ, Harrison TW. Is it time to move away from short-acting beta-agonists in asthma management? Eur Respir J 2019;53.

55 Axelsson M, Emilsson M, Brink E, et al. Personality, adherence, asthma control and health-related quality of life in young adult asthmatics. Respir Med 2009;103:1033-40.

56 Bender B, Zhang L. Negative affect, medication adherence, and asthma control in children. J Allergy Clin Immunol 2008;122:490-5.

57 Davidsen JR, Hallas J, Søndergaard J, et al. Association between prescribing patterns of anti-asthmatic drugs and clinically uncontrolled asthma: a cross-sectional study. Pulm Pharmacol Ther 2011;24:647-53

58 Kitch BT, Paltiel AD, Kuntz KM, et al. A single measure of FEV 1 is associated with risk of asthma attacks in long-term follow-up. Chest 2004;126:1875-82.

59 Tan X, Patel I, Chang J. Review of the four item Morisky medication adherence scale (MMAS-4) and eight item Morisky medication adherence scale (MMAS-8). Inov Pharm 2014;5:165.

60 Horne R, Weinman J, Hankins M. The beliefs about medicines questionnaire: the development and evaluation of a new method for assessing the cognitive representation of medication. Psychol Health 1999;14:1-24.

61 Hahn SR, Park J, Skinner EP, et al. Development of the ASK-20 adherence barrier survey. Curr Med Res Opin 2008;24:2127-38.

62 McNutt L-A, Wu C, Xue X. Estimating the relative risk in cohort studies and clinical trials of common outcomes. Am J Epidemiol 2003;157:940-3. 